\title{
Versión española del Sport Satisfaction Instrument (SSI) adaptado al aprendizaje de la Educación Física bilingüe en Inglés
}

\author{
Antonio BAEnA-ExTREMERA \\ Universidad de Murcia \\ Antonio Granero-Gallegos \\ Consejería de Educación de la Junta de Andalucía
}

Recibido: 12 febrero 2014 / Aceptado: 12 agosto 2014

ISSN: 1697-7467

\begin{abstract}
RESUMEN: El objetivo de esta investigación ha sido analizar las propiedades psicométricas del Cuestionario de Satisfacción hacia la Educación Física Bilingüe. Con ello se pretendió determinar si constituye un instrumento válido y fiable para su utilización en futuras investigaciones. Se administró a un total de 328 estudiantes de secundaria de 12 a 16 años, analizándose la estructura del instrumento mediante procedimientos confirmatorios. Este análisis apoya el modelo teórico hipotetizado de dos factores (satisfacción/diversión y aburrimiento), que mantienen correlaciones negativas entre sí. El instrumento creado mostró niveles aceptables de consistencia interna y estabilidad temporal como para poder ser utilizado. Palabras clave: bilingüismo, satisfacción, diversión, aburrimiento, propiedades psicométricas, SSI-EF, educación bilingüe, inglés.
\end{abstract}

Spanish Version of the Sport Satisfaction Instrument (SSI) Adapted to Learning Bilingual in English Physical Education

ABSTRACT: The aim of this research was to analyze the psychometric properties of the Satisfaction Questionnaire to Bilingual Physical Education. This was intended to determine whether it constitutes a valid and reliable instrument to be used in future researches. The scale was administered to 328 high school students from 12 to 16 years old. The structure of the instrument was analyzed by confirmatory procedures. This analysis supports the hypothesized theoretical model of two factors (satisfaction/fun and boredom), maintaining negative correlations each other. The instrument introduced showed acceptable levels of internal consistency and temporal stability to be able to be used.

Keywords: bilingualism, satisfaction, fun, boredom, psychometric properties, SSI-PE, bilingual education, English.

\section{INTRODUCCIÓN}

La enseñanza de los idiomas en los centros educativos, tanto de primaria como secundaria, está en continuo cambio. Hoy por hoy, la tendencia general de estos centros es a impartir una enseñanza bilingüe e, incluso, trilingües. En el caso de la Comunidad Autónoma de Andalucía, siguiendo la Orden 28 de Junio de 2011 por la que se regula la enseñanza 
bilingüe en los centros docentes de esta comunidad, se especifica que al menos dos áreas no lingüísticas han de ser enseñadas parcialmente en lengua extranjera (LE). A partir de aquí, la autonomía pedagógica y organizativa de los centros permite, según criterios pedagógicos, que cada centro pueda seleccionar las materias a enseñar a través de una lengua extranjera. En este punto, la Educación Física (EF) adquiere una importante función, por las implicaciones educativas que conlleva y las particularidades de esta área para su aprendizaje (Coral i Mateu, 2011; Fernández, 2011; Montálvez, López, \& Mariscal, 2002).

Una de las principales preocupaciones en la adquisición de una segunda lengua o LE ha sido la motivación del alumnado hacia el aprendizaje de este idioma (Khodadady \& Khajavy, 2012), pues esta variable ha sido ampliamente aceptada por los docentes e investigadores como uno de los factores clave que influyen en el éxito del aprendizaje de una LE (Ghazvini \& Khajehpour, 2011). Sobre esto, Gardner (1985) define la motivación en la LE como el grado en que una persona realiza trabajos individuales y se esfuerza por aprender la lengua, debido fundamentalmente al deseo de hacerlo bien y de sentirse satisfecho con la experiencia de esta actividad. Tanto la motivación como la satisfacción son variables que están estrechamente ligadas a algunas de las teorías psicológicas más estudiadas en estos años en el aprendizaje de los idiomas, como es la Teoría de la Autodeterminación, ya utilizada en el trabajo de Khodadady y Khajavy (2012) entre otros. Por ejemplo, Casas, Baltatescu, Bertran, González, \& Hatos (2013) encontraron en su investigación una importante y significativa relación entre la satisfacción y la motivación académica.

En el caso de la satisfacción, los investigadores creen que es una variable importante y que preocupa a los docentes de EF e idiomas, ya que unos de los objetivos de la enseñanza es que a la vez que el alumno aprende, se divierta y esté satisfecho con los conocimientos que adquiere. Pero además, la satisfacción tiene connotaciones determinantes en el proceso de enseñanza-aprendizaje del alumnado, como veremos. Por ejemplo, se ha podido comprobar que la satisfacción que los estudiantes sienten hacia la escuela se relaciona con los niveles de satisfacción de la vida de los estudiantes (Huebner, Valois, Paxton, \& Drane, 2005), el estrés (Hui \& Sun, 2010), las relaciones sociales entre sus iguales (Skinner, Furrer, Marchand, \& Kinderman, 2008) y con el rendimiento académico (Huebner \& Gilman, 2006), determinando el compromiso del estudiante con su trabajo escolar (Danielsen, Breivik, \& Wold, 2011). Pero si estas razones no fueran suficientes para apreciar la importancia de esta variable, otros autores, como Busse y Walter (2013), hablan de una estrecha relación significativa entre la satisfacción y el compromiso de los estudiantes que deciden aprender un nuevo idioma; aspecto que deberíamos aprovechar como docentes.

Por el contrario, los alumnos insatisfechos se han asociado con diversos comportamientos negativos, como el absentismo escolar y la depresión (Luopa, Pietikäinen, \& Jokela, 2006) llegando incluso a influir en el abandono escolar (Takakura, Bake, \& Kobayashi, 2010). Pero además, estudios recientes sugieren que tales conductas negativas del estudiante influyen también en los profesores, generando altos niveles de estrés (Geving, 2007). Es más, algo que preocupa, y del cual ya se pueden encontrar algunos trabajos de investigación, es del estrés o ansiedad que sufren los estudiantes en el aprendizaje de una LE (Khodadady \& Khajavy, 2012; Kirova, Petkovska, \& Koceva, 2012). Por todo ello, se aprecia la importancia que puede tener para el aprendizaje de un idioma, conocer y poder trabajar para conseguir en el alumnado una importante satisfacción hacia el aprendizaje de una LE a través de otras materias, como es la EF. 
Para medir el grado de satisfacción se han creado, a día de hoy, pocos instrumentos fiables que la puedan medir. Nicholls, Cheung, Lauer y Patashnick (1989) y Nicholls, Patashnick y Nolen (1985), elaboraron la Intrinsic Satisfaction Classroom Scale (ISC) para medir el grado de satisfacción del alumno con respecto al ámbito escolar. Estos autores llevaron a cabo un trabajo con 587 escolares de diversos centros educativos, creando un instrumento que medía el grado de satisfacción y de interés intrínseco de los estudiantes hacia la escuela, a través de ocho ítems divididos en dos escalas, que miden la satisfacción/diversión (cinco ítems) y el aburrimiento (tres ítems) en la práctica de actividades académicas.

Algunos años después, y en el campo de la EF y el deporte, Duda y Nicholls (1992) realizaron una investigación con 207 estudiantes de secundaria, en la que utilizaron la ISC adaptándola al deporte, modificando los ítems paralelamente y surgiendo la Sport Satisfaction Instrument (SSI). El análisis factorial de esta versión reveló la existencia de dos factores al igual que la escala de origen, que incluyen tres ítems para el factor de aburrimiento $(\alpha=$ .71), y cinco ítems para el factor de satisfacción/diversión $(\alpha=.82)$.

Recientemente, Baena-Extremera, Granero-Gallegos, Bracho-Amador y Pérez-Quero (2012) adaptaron la SSI de deporte a la EF en el contexto español, dando lugar a la SSI-EF. Esto autores obtuvieron resultados similares a los trabajos previos y demostraron la validez de dicha escala en el citado contexto. A pesar de los trabajos nombrados, las investigaciones relacionadas con la satisfacción y con la satisfacción hacia la EF son muy escasos, tanto dentro como fuera de nuestro país (Baena-Extremera et al., 2012). Pero lo más importante de todo es que en la literatura existente apenas se encuentran trabajos que midan el grado de satisfacción en el aprendizaje de una LE en los escolares y menos en EF, por lo que los resultados de este estudio serían de gran interés para la comunidad educativa y científica.

Teniendo en cuenta lo expuesto y debido a que hoy por hoy la EF se está enseñando en un segundo idioma (sobre todo en inglés) en gran número de centros educativos, el objetivo de este trabajo es aportar evidencias sobre la dimensionalidad de la versión española del SSI-EF en una muestra de adolescentes estudiantes de educación secundaria bilingüe, utilizando procedimientos confirmatorios.

\section{MÉTODo}

\subsection{Participantes}

El diseño muestral fue de tipo no probabilístico y por conveniencia, participando un total de 328 alumnos $(153$ chicos $=46.7 \% ; 175$ chicas $=53.3 \%)$ de 12 centros de Educación Secundaria de las provincia de Almería, Córdoba, Granada y Jaén. El rango de edad estuvo comprendido entre 12 y 16 años [media $(M)=13.37$; desviación típica $(D T)=1.40$ ]. La distribución por cursos fue la siguiente: $30.6 \%$ estudian $1^{\circ}$ de ESO; $21.2 \%, 2^{\circ}$ de ESO; $25.4 \%, 3^{\circ}$ de ESO; y $22.8 \%, 4^{\circ}$ de ESO.

Para evaluar la estabilidad temporal del SSI-EF adaptado a la EF en inglés se utilizó una segunda muestra de 125 alumnos, elegida previamente de forma aleatoria simple y compuesta por 60 chicos $(M=13.02 ; D T=1.35)$ y 65 chicas $(M=13.95 ; D T=1.40)$ que completaron nuevamente el instrumento siete semanas más tarde. Con el objeto de prevenir posibles tendencias de deseabilidad social, los adolescentes fueron instruidos para que utilizaran su fecha de nacimiento para identificar sus cuestionarios. 


\subsection{Instrumentos}

Al contrario que en el trabajo de Franco, Pino y Rodríguez (2012), no se partió de la construcción desde el inicio de un cuestionario nuevo a raíz de aportaciones de otros trabajos, sino que se partió de un instrumento ya reconocido por la comunidad científica como válido y fiable, que serviría de base y el cual sería adaptado para el propósito de esta investigación. Concretamente, el instrumento es el Sport Satisfaction Instrument (SSI), utilizándose la versión validada al contexto español y adaptada a la EF (SSI-EF) por BaenaExtremera et al. (2012), del original Sport Satisfaction Instrument (SSI) (Duda \& Nicholls, 1992). Este instrumento presenta ocho ítems que miden el grado de satisfacción en las actividades de EF, con dos subescalas que miden la satisfacción/diversión (SAT/D) (cinco ítems) y el aburrimiento (ABU) (tres ítems). Las respuestas se recogieron en una escala de ítems politómicos que oscilaba entre 1 (totalmente en desacuerdo) y 5 (totalmente de acuerdo).

\subsection{Proceso de adaptación}

Se adaptó la versión española del SSI-EF (Baena-Extremera et al., 2012), al ser su objeto de estudio la EF y el nuestro la EF bilingüe. La evaluación cualitativa de ítems ( $v a-$ lidez de contenido) se efectuó mediante el juicio de cuatro expertos (Osterlind, 1989): dos en construcción de escalas y dos conocedores del constructo a evaluar. Se les entregó una tabla de especificaciones de los ítems (Calabuig \& Crespo, 2009; Spaan, 2006), en la que se recogía la definición semántica del constructo a evaluar y la de su componente. Se les mostró el listado de ítems tras la adaptación de los originales. Tenían que emitir un juicio sobre su pertinencia y su compresión en escala de 1 (muy en desacuerdo) a 4 (muy de acuerdo). Además, disponían de un apartado en el que hacer las anotaciones y observaciones generales sobre cada uno de los ítems, pudiendo realizar una redacción alternativa de cada ítem si lo consideraban conveniente. Aquellos ítems que obtuvieron puntuaciones medias $<2.5$, tanto en pertinencia como en comprensión, fueron revisados. Si el ítem no era clasificado por al menos tres de los cuatro expertos dentro de las dimensiones teóricas (satisfacción/diversión y aburrimiento), era nuevamente revisado, analizando posibles problemas antes de proponer una redacción alternativa que recogiese la dimensión teórica de forma más clara y precisa. El encabezamiento fue: "Indica tu grado de desacuerdo o acuerdo con las siguientes afirmaciones, referidas a tus clases de Educación Física en inglés". La concordancia global de los cuatro expertos sobre la pertinencia y comprensión de los ítems se midió mediante el Coeficiente de Correlación Intraclase (CCI), a partir de un modelo de efectos mixtos y asumiendo una definición de acuerdo absoluto; los valores obtenidos fueron: $\mathrm{CCI}=.70$ en pertinencia y $\mathrm{CCI}=.85$ en comprensión.

Además, para medir la dispersión en el acuerdo de los jueces, se utilizó como criterio el recorrido intercuartílico. De modo que, si la diferencia del percentil Q3 frente al percentil Q1 era igual a 0 o 1, el ítem se aceptaba y/o modifica ligeramente; si dicha diferencia se situaba entre 1 y 2 , se revisaba y reformulaba el ítem; mientras que si era superior a 2, se entendía que la dispersión era tan alta entre los juicios de los jueces que el ítem era rechazado. En la tabla 1, se expresan los datos de pertinencia y comprensión de los jueces. 
Tabla 1. Rangos intercuartílicos

\begin{tabular}{|l|c|c|c|c|c|c|}
\hline & \multicolumn{3}{|c|}{ Comprensión } & \multicolumn{3}{c|}{ Pertinencia } \\
\cline { 2 - 7 } & $Q 3$ & $Q 1$ & $\begin{array}{c}\text { Rango } \\
\text { Interc. }\end{array}$ & Q3 & $Q 1$ & $\begin{array}{c}\text { Rango } \\
\text { Interc }\end{array}$ \\
\hline Juez 1 & 3 & 2 & 1 & 3 & 2 & 1 \\
\hline Juez 2 & 3 & 2 & 1 & 4 & 4 & 0 \\
\hline Juez 3 & 3 & 3 & 0 & 3 & 2 & 1 \\
\hline juez 4 & 2 & 2 & 0 & 3 & 2 & 1 \\
\hline
\end{tabular}

Finalmente, los comentarios de los mismos sobre instrucciones y forma de redacción supusieron cambios menores. La nueva versión fue administrada a 70 alumnos de educación secundaria con edades entre 12 y 16 años. Tras el análisis de los resultados psicométricos obtenidos ( $\alpha=.79$ en satisfacción/diversión y $\alpha=.70$ en aburrimiento), y una última revisión por parte del equipo de investigación se llegó a la versión final española del SSI adaptado a la EF bilingüe (SSI-PE).

\subsection{Procedimiento}

Se obtuvieron los permisos necesarios para realizar la investigación por parte de los órganos de dirección de los centros educativos bilingües en los que algunas clases de EF de las líneas bilingües se impartían en inglés. En primer lugar se habló con los equipos directivos de los centros y se solicitó informes, previo permiso de los docentes, de la habilitación del profesorado en inglés. Se requirió un nivel mínimo de B1 entre el profesorado que impartía las clases de EF. Asimismo, se concertó una reunión previa con los docentes de cada centro en la que se les explicó el objetivo del estudio y la necesidad de que impartieran de forma habitual la clase de EF en inglés (en su totalidad o en gran parte de la duración de la clase). Entre las preguntas iniciales del cuestionario, referentes a los datos sociodemográficos de los participantes se añadió una pregunta filtro de respuesta dicotómica (sí/no) para que respondieran si realmente las clases de EF se impartían en su mayoría de duración en inglés. Así, de los 567 cuestionarios recogidos, 328 son los que realmente se utilizaron para esta investigación al responder afirmativamente a esta cuestión, pues los 239 restantes respondieron que no era habitual que las clases se impartieran en inglés. Los estudiantes fueron informados del propósito del estudio y de sus derechos como participantes en el mismo. Los instrumentos para medir las diferentes variables se administraron en el aula, sin la presencia del docente. Cada participante tuvo entre 10-20 minutos para completar los cuestionarios. Las respuestas al instrumento se mantendrán en el anonimato.

\subsection{Análisis estadísticos}

Dado que las estructuras que subyacen al instrumento analizado han sido consistentemente determinadas en la literatura ya citada, para evaluar la estructura factorial de la escala se realizó un Análisis Factorial Confirmatorio (AFC) con LISREL 8.80. Los análisis de ítems, 
homogeneidad y estructura interna y consistencia interna (alfa de Cronbach) de la escala, se realizaron con el SPSS 17.0.

\section{Resultados}

\subsection{Análisis de ítems y fiabilidad de la escala}

En la Tabla 2 se muestran los estadísticos descriptivos del SSI-PE. En el análisis estadístico de los ítems se mantuvo la distribución ítem-factor observada en el instrumento original (Baena-Extremera et al., 2012; Duda \& Nicholls, 1992). Las características de los ítems fueron analizadas comprobando si el alfa de la escala aumentaba con la eliminación del algún ítem, así como teniendo en cuenta los criterios apuntados por Nunnally y Bernstein (1995) para conservar un ítem dentro de un factor: coeficiente de correlación corregido ítem-total $(C C I T-c) \geq .30$, desviación típica $(D T)>1$, y que todas las opciones de respuesta habían sido usadas en algún momento. Como se puede comprobar, y según las recomendaciones de Bollen y Long (1994), los índices de asimetría y curtosis son próximos a 0 y < 2 en los dos factores.

Los ítems del primer factor (satisfacción/diversión) presentaron valores medios entre 3.61 del ítem 5 y 4.02 del ítem 8. Las $D T$ fueron $>1$, oscilando entre 1.06 del ítem 1 y 1.16 del ítem 5. La consistencia interna de esta dimensión fue adecuada $(\alpha=.83)$. Todos los CCIT-c presentaron valores superiores a .60 .

El segundo factor (aburrimiento) presentó ítems con valores medios entre 2.06 para el ítem 4 y 2.33 del ítem 2. Las $D T$ fueron $>1$, oscilando entre 1.23 del ítem 2 y 1.32 del ítem 4. La consistencia interna de esta dimensión fue adecuada $(\alpha=.71)$. Todos los CCIT-c presentaron valores $>$.43. A pesar de estos datos y de la adecuada consistencia interna obtenida, los resultados arrojan que con la eliminación del ítem 2 de esta dimensión se alcanzaría un alfa .73, al igual que ya ocurría en el trabajo de Baena-Extremera et al. (2012), aunque solo se mejora un .02 respecto a la fiabilidad de los tres ítems, se decide tener en cuenta para el análisis del modelo.

Autores como Carretero-Dios y Pérez (2007) y Clark y Watson (2003) recomiendan realizar un estudio de correlación para asegurar la homogeneidad de cada dimensión. En el presente trabajo, la correlación entre la puntuación de cada ítem y la puntuación total en cada uno de los componentes muestran correlaciones positivas con la dimensión teórica a la que pertenecen y negativa con la puntuación total de la otra dimensión. También en este caso se llama la atención en relación al ítem 2, pues se puede observar cómo obtuvo el valor negativo más cercano a cero $(\mathrm{r}=-.23)$; no obstante, no muestra solapamiento con la dimensión teórica de satisfacción/diversión. 
Tabla 2. Estadísticos descriptivos, de consistencia interna y de homogeneidad.

\begin{tabular}{|c|c|c|c|c|c|c|c|}
\hline Escala: & $M$ & $D T$ & CCIT-c & $C C$ & $\begin{array}{l}\alpha \sin \\
\text { item }\end{array}$ & Asimetria & Curtosis \\
\hline \multicolumn{8}{|l|}{$\begin{array}{l}\text { Satisfacción/diversión en EF } \\
\text { bilingüe }(\alpha=.83)\end{array}$} \\
\hline $\begin{array}{l}1 \text { Normalmente me divierto } \\
\text { en las clases de Educación } \\
\text { Física en inglés }\end{array}$ & 3.99 & 1.06 & .68 & -.47 & .84 & -.97 & -.40 \\
\hline $\begin{array}{l}5 \text { Normalmente encuentro } \\
\text { la Educación Física en } \\
\text { inglés interesante }\end{array}$ & 3.61 & 1.16 & .71 & -.39 & .83 & -.63 & .02 \\
\hline $\begin{array}{l}6 \text { Cuando hago Educación } \\
\text { Física en inglés parece } \\
\text { que el tiempo vuela }\end{array}$ & 3.98 & 1.21 & .69 & -.45 & .83 & -1.00 & .17 \\
\hline $\begin{array}{l}7 \text { Normalmente participo } \\
\text { activamente en las clases } \\
\text { de Educación Física en } \\
\text { inglés }\end{array}$ & 3.95 & 1.08 & .62 & -.38 & .85 & -.91 & .39 \\
\hline $\begin{array}{l}8 \text { Normalmente me lo paso } \\
\text { bien haciendo Educación } \\
\text { Física en inglés }\end{array}$ & 4.02 & 1.12 & .75 & -.46 & .82 & -1.01 & -.40 \\
\hline \multicolumn{8}{|l|}{$\begin{array}{l}\text { Aburrimiento en EF bilingüe } \\
(\alpha=.71)\end{array}$} \\
\hline $\begin{array}{l}2 \text { En las clases de } \\
\text { Educación Física en } \\
\text { inglés a menudo sueño } \\
\text { despierto en vez de } \\
\text { pensar en lo que hago } \\
\text { realmente }\end{array}$ & 2.33 & 1.23 & .44 & -.23 & .73 & .55 & -.72 \\
\hline $\begin{array}{l}3 \text { En las clases de } \\
\text { Educación Física en } \\
\text { inglés, normalmente me } \\
\text { aburro }\end{array}$ & 2.10 & 1.26 & .62 & -.49 & .53 & .87 & -.37 \\
\hline $\begin{array}{l}4 \text { En Educación Física en } \\
\text { inglés deseo que la clase } \\
\text { termine rápidamente }\end{array}$ & 2.06 & 1.32 & .56 & -.52 & .60 & .89 & -.55 \\
\hline
\end{tabular}




\subsection{Análisis factorial confirmatorio}

Con el objeto de confirmar la dimensionalización original propuesta teóricamente (Baena-Extremera et al., 2012; Duda \& Nicholls, 1992), se aplicaron modelos de ecuaciones estructurales. La estructura factorial del instrumento se evaluó con AFC. Este análisis se llevó a cabo utilizando el método de estimación weighted least squares (WLS) para variables ordinales del programa LISREL 8.80 (Jöreskog \& Sörbom, 2003). La matriz de correlaciones policóricas y la matriz de covarianzas asintóticas fueron utilizadas como input para el análisis de los datos. Se hipotetizó un modelo de medida, consistente en un modelo de dos factores que asumió la existencia de dos variable latentes.

Teniendo en cuenta que se desaconseja utilizar una única medida de ajuste global del modelo, se calcularon varios índices de ajuste, como recomiendan, entre otros, autores como Bentler (2007), Markland (2007) o Miles y Shevlin (2007). Por ello, el ajuste del modelo fue evaluado con una combinación de índices de ajuste absolutos y relativos. Entre los absolutos, se utilizó el valor $p$, asociado con el estadístico chi cuadrado $\left(c^{2}\right)$, que prueba el modelo nulo frente al modelo hipotetizado (Barrett, 2007). La ratio entre $c^{2}$ y grados de libertad $(g l)\left(\chi^{2} / g l\right)$ es un heurístico que se utiliza para reducir la sensibilidad del $c^{2}$ al tamaño de la muestra. En un modelo considerado perfecto su valor sería de 1.0 y las ratios por debajo de 2.0 se considerarán como indicadores de muy buen ajuste del modelo (Tabachnik \& Fidell, 2007), mientras que valores por debajo de 5.0 son considerados aceptables (Hu \& Bentler, 1999). Asimismo, se ha calculado el GFI (índice de bondad de ajuste), que indica la cantidad relativa de varianza y covarianza reproducida por el modelo específico, comparado con el modelo saturado, y cuyo valor debe ser igual o superior a .90 para considerar mínimamente aceptable el ajuste de un modelo, aunque autores como Hooper, Coughlan y Mullen (2008) consideran valores $\geq .95$ para un mejor ajuste. Entre los índices relativos se ha utilizado el NFI (índice de ajuste normalizado), el NNFI (índice de ajuste no normativo) y CFI (índice de ajuste comparativo). En los índices incrementales se considera que valores $\geq .95$ indican un buen ajuste (Hu \& Bentler, 1999). Autores como Kline (2005) recomiendan la utilización de RMSEA (error de aproximación cuadrático medio) y, según Hu y Bentler (1999) un valor $\leq .06$ indicaría un buen ajuste. Los parámetros estimados se consideran significativos cuando el valor asociado al valor $t$ es superior a $1.96(p<.05)$.

Atendiendo a las recomendaciones de autores como Markland (2007) o Levy y Hancock (2007), de formular y analizar varios modelos si los datos así lo recomiendan y de reportar los resultados más relevantes, se presentan, a continuación, los resultados del AFC correspondientes a dos modelos: uno con 8 ítems y otro con 7 ítems. El modelo con 7 ítems se realizó sin el ítem-2, teniendo en cuenta lo expuesto con anterioridad en este trabajo en relación con la consistencia interna y la homogeneidad de los ítems (Tabla 2) y tomando en consideración que este ítem ha sido eliminado del factor aburrimiento en trabajos como el de Castillo, Balaguer y Duda (2001).

Los índices de bondad de ajuste obtenidos en el modelo de 8 ítems mostraron un ajuste satisfactorio: $c^{2}=20.321, g l=13, p=.087, c^{2} / g l=1.56, G F I=.953, N F I=.989, N N F I$ $=.986, C F I=.991, R M S E A=.047$. Respecto al modelo de 7 ítems, aunque los índices relativos mostraron un buen ajuste, los resultados de los índices absolutos recomendaron 


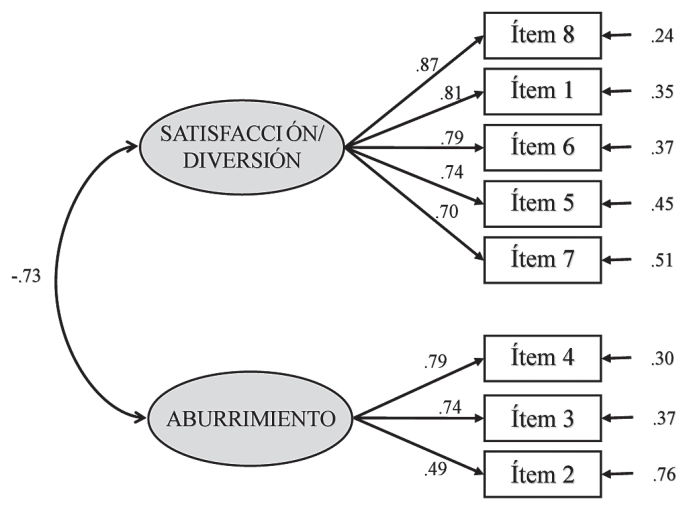

Figura 1. Path Diagram del AFC, con pesos estandarizados y errores de medición de cada uno de los items de la versión española del SSI adaptado a la Educación Física bilingüe. Modelo 8 items.

rechazar el modelo, atendiendo las recomendaciones de autores como Barrett (2007), pues la prueba $c^{2}$ resultó significativa: $c^{2}=33.993, g l=19, p=.018, c^{2} / g l=1.79, G F I=.964$, $N F I=.982, N N F I=.987, C F I=.978, R M S E A=.056$

La estabilidad temporal se evaluó con la citada muestra de 125 alumnos que completaron el SSI-PE en dos ocasiones con intervalo de siete semanas. Los resultados del pretest en satisfacción/diversión fueron $\alpha=.90$ y en aburrimiento, $\alpha=.81$. Los datos del postest en satisfacción/diversión fueron $\alpha=.93$ y en aburrimiento, $\alpha=.80$. Los valores de correlación test-retest en la dimensión satisfacción/diversión fueron $r=.81$ y en aburrimiento $r=.79$.

\section{Discusión}

El plurilingüismo es hoy en día una necesidad tanto en la enseñanza primaria como en la secundaria, llegando incluso a ser necesario para el acceso a algunos estudios universitarios (Toledo, Rubio, \& Hermosín, 2012). Por eso es fundamental que la enseñanza de otros idiomas comience cuanto antes a través del bilingüismo con otras materias, y que éste aprendizaje, pueda ser controlado por el docente y las instituciones. Una de las formas de poder establecer este control, es midiendo el grado de satisfacción del alumno en el aprendizaje de una LE.

Entre las asignaturas bilingües que se imparten en los centros educativos españoles, la EF es una de las preferidas por los estudiantes, al igual que ocurre en otros países (Juriševič \& Pižorn, 2013). En esta asignatura, las posibilidades de interacción entre el alumnado y el profesor (Baena-Extremera \& Ruiz, 2009), el tipo de tareas, la actividad motriz y cognitiva requerida a los alumnos (Skutnabb-Kangas \& Dunbar, 2010), la desinhibición y la reducción del estrés y ansiedad con la catarsis del ejercicio (McGuirre, Parker, \& Cooper, 2001), hacen de la EF una materia importante en el aprendizaje de LE en el entorno escolar. Por ello, un aspecto que resultaría de gran ayuda, sería conocer el grado de satisfacción del alumnado 
hacia la EF bilingüe pues, como se ha especificado en la introducción de este trabajo, la satisfacción presenta connotaciones educativas muy importantes, entre ellas, el compromiso con las tareas académicas (Skinner et al. 2008), la reducción del abandono escolar (Elmore \& Huebner, 2010), la mejora en las calificaciones y en los problemas de conducta (ver Baker \& Maupin, 2009; Huebner, Gilman, Reschly, \& Hall , 2009).

Como se ha destacado, apenas existen investigaciones que evalúen el grado de satisfacción del alumnado en las diferentes materias, al menos en el caso de las asignaturas bilingües. Por ello, el objetivo principal de esta investigación ha sido validar en una muestra de estudiantes de centros bilingües, la adaptación del SSI-EF a la EF bilingüe, dando como resultado la escala SSI-PE.

La utilidad de este instrumento es importantísima, porque ayudaría, además, a conocer el grado de satisfacción del alumnado ya que, según Busse y Walter (2013), los estudiantes insatisfechos participan menos en las tareas de clase e, incluso, comienzan a evitar las clases en otro idioma. Pero además, teóricamente, cuanto mayor sea el grado de satisfacción, mayor será también el de motivación y, por tanto, menor sería la ansiedad en el aprendizaje del idioma (Khodadady \& Khajavy, 2012). Esto es interesante porque diversos investigadores demuestran que para algunos alumnos el aprendizaje de una LE puede ser una actividad estresante (Hewitt \& Stefenson, 2011) y el estrés en el aprendizaje de LE conlleva una importante desmotivación en el alumnado (Liu \& Huang, 2011).

\section{Conclusiones y Limitaciones}

Tras llevar a cabo los análisis psicométricos necesarios y su correspondiente AFC basado en modelos de ecuaciones estructurales, los resultados apoyan la validez factorial y la fiabilidad del instrumento adaptado en dos dimensiones (satisfacción/diversión y aburrimiento), similares al del modelo hipotetizado de origen, y coincidente con los trabajos señalados.

Los datos confirman la correlación significativa y negativa obtenida entre ambos factores, demostrando su independencia, tal como ocurre en el resto de investigaciones señaladas. Igualmente, la consistencia interna es aceptable en ambas dimensiones, situándose éstos valores dentro del margen válido y por encima del encontrado en la línea de investigaciones similares. Además, los ítems tienen una correlación elevada con su factor correspondiente y baja con los del otro. A pesar de ello, con la eliminación del ítem 2 de aburrimiento se incrementaría ligeramente el alfa de Cronbach (de .71 a .73). Teniendo en cuenta además que Castillo et al. (2001) en deporte eliminaron el citado ítem para llegar a una aceptable fiabilidad del factor, en este trabajo se analizó un modelo de ecuaciones estructurales con 7 ítems y con 8 para poder hacer comparaciones. Aunque finalmente el modelo de 8 ítems resultó más adecuado, como ocurría en la versión de EF de Baena-Extremera et al. (2012), estos resultados se han de tener en cuenta para futuras investigaciones sobre la EF bilingüe en las que el modelo de 7 ítems puede resultar con un buen ajuste.

Los análisis psicométricos efectuados a la escala SSI-PE demuestran que las puntuaciones que se obtienen cuando se aplica el mismo son válidas y fiables. Así pues, es de destacar la gran utilidad que presenta este instrumento para los docentes, tanto en relación con el área como con la adherencia al aprendizaje de otro idioma, en este caso, el inglés. 
Para finalizar, una de las limitaciones que podríamos destacar de esta investigación es el haber realizado un diseño muestral para Andalucía, pero por limitaciones económicas y temporales se decidió centrar el estudio en centros educativos bilingües de las provincias mencionadas, utilizando una muestra seleccionada al azar. Una posible opción para futuros estudios podría centrarse en el análisis del funcionamiento diferencial de ítems (DIF) contrastando la equivalencia entre diferentes submuestras de estudiantes, tanto de primaria como de secundaria, de centros públicos y privados, entre diferentes provincias y comunidades e, incluso, entre diferentes idiomas. También, podría crearse una línea de investigación centrada en la satisfacción del alumnado bilingüe tras la aplicación de diversos programas de intervención en EF, como el llevado a cabo por Cepero, García y López (2013). A pesar de la limitación de la muestra por no ser significativa por ejemplo en una comunidad, una de las fortalezas más destacable de este trabajo es el tamaño de la misma, al tratarse de clases bilingües. Se trata de una muestra suficientemente amplia, que le otorga al trabajo calidad y representatividad más que necesarias.

Esta investigación ha permitido discutir los trabajos apenas ya existentes. Asimismo, una de las fortalezas más destacables es el resultado obtenido. Hay que resaltar que se ha trabajado con una escala reconocida y probada a nivel internacional en EF, y su validación para EF bilingüe en español supone una contribución al desarrollo de nuevas vías de investigación y apoyo a las ya existentes.

\section{REFERENCIAS}

Baena-Extremera, A., Granero-Gallegos, A, Bracho-Amador, C., y Pérez-Quero, F. J. (2012). "Versión española del Sport Satisfaction Instrument (SSI) adaptado a la Educación Física", en Revista de Psicodidáctica, 17(2): 377-396.

Baena-Extremera, A., y Ruiz, P. (2009). "Tratamiento educativo de la coeducación y la igualdad de sexo en el contexto escolar y en especial en Educación Física", en Aula Abierta, 37(2): 111-122.

Baker, J. A. y Maupin, A. N. (2009). School satisfaction and children's positive school adjustment. En R. Gilman, E. S. Huebner, \& M. J. Furlong (Eds.), Handbook of positive psychology in the schools. New York: Routledge, 189-196.

Barrett, P. (2007). "Structural equation modelling: Adjudging model fit", en Personality and Individual Differences 42: 815-824.

Bentler, P. M. (2007). "On tests and indices for evaluating structural models", en Personality and Individual Differences 42: 825-829.

Bollen, K. A. y Long, J. (1994). Testing structural equation models. Newbury Park, CA: Sage.

Busse, V. y Walter, C. (2013). "Foreign Language Learning Motivation in Higher Education: A Longitudinal Study of Motivational Changes and Their Causes", en The Modern Language Journal, 97(2): 435-456.

Calabuig, F. y Crespo, J. (2009). "Uso del método Delphi para la elaboración de una medida de la calidad percibida de los espectadores de eventos deportivos", en Retos: Nuevas tendencias en Educación Física, Deporte y Recreación, 16: 21-25.

Carretero-Dios, H. y Pérez, C. (2007). "Standards for the development and the review of instrumental studies: Considerations about test selection in psychological research", en International Journal of Clinical and Health Psychology, 7: 863-882. 
Casas, F., Baltatescu, S., Bertran, I., González, M. y Hatos, A. (2013). “School Satisfaction Among Adolescents: Testing Different Indicators for its Measurement and its Relationship with Overall Life Satisfaction and Subjective Well-Being in Romania and Spain”, en Social Indicators Resesearch, 111: 665-681.

Castillo, I., Balaguer, I. y Duda, J.L. (2001). "Perspectivas de meta de los adolescentes en el contexto académico", en Psicothema, 13(1): 79-86.

Cepero, M., García, A. y López, M. (2013). "Diseño de un Programa de intervención bilingüe para el área de Educación Física basado en la competencia digital”, en Porta Linguarum, 19: $257-273$.

Clark, L. A. y Watson, D. (2003). Constructing validity: Basic issues in objective scale development. En A. E. Kazdin (Ed.), Methodological issues \& strategies in clinical research (3 ${ }^{\mathrm{a}}$ ed.). Washington: APA, 207-231.

Coral i Mateu, J. (2011). "Mou-te i aprèn. Aprenentatge integrat de continguts d'educació física, salut i llengua anglesa. CLIL in Physical Education. En C. Escobar Urmeneta, AICLE-CLILEMILE Educació plurilingüe: Experiencias, research \& politiques. Barcelona: Universitat Autónoma de Barcelona. Servei de Publicacions, 181-192.

Danielsen, A. G., Breivik, K. y Wold, B. (2011). "Do Perceived Academic Competence and School Satisfaction Mediate the Relationships Between Perceived Support Provided by Teachers and Classmates, and Academic Initiative?", en Scandinavian Journal of Educational Research, 55(4): 379-401.

Duda, J. L. y Nicholls, J. G. (1992). "Dimensions of achievement motivation in schoolwork and sport”, en Journal of Educational Psychology, 84(3): 290-299.

Elmore, G. M. y Huebner, E. S. (2010). "Adolescents'satisfaction with school experiences: relationships with demographisc, attachment relationships, and school engagement behavior", en Psychology in the Schools, 47(6): 525-537.

Fernández, E. (2011). "El valor del realismo en las tareas de educación física bilingüe”, en Revista Digital Educativa Wadi-red (1).

Franco, P., Pino, M. y Rodríguez, B. (2012). "Características psicométricas de un cuestionario en español para medir estrategias de aprendizaje del inglés como lengua extranjera", en Porta Linguarum, 18: 27-42.

Gardner, R. (1985). Social psychology and second language learning. London: Edward Arnold.

Ghazvini, S. D. y Khajehpour, M. (2011). "Attitudes and Motivation in learning English as Second Language in high school students", en Procedia-Social and Beavioral Sciences, 15: 1209-1213.

Geving, A. M. (2007). "Identifying the types of student and teacher behaviours associated with teacher stress", en Teaching and Teacher Education, 23: 624-40.

Hewitt, E. y Stephenson, J. (2011). "Foreign language anxiety and oral exam performance: A replication of Phillips's MLJ study", en The Modern Language Journal, 96: 170-189.

Hooper, D., Coughlan, J. y Mullen, M. (2008). "Structural Equation Modelling: Guidelines for Determining Model Fit", en Electronic Journal of Business Research Methods, 6(1): 53-60.

Hu, L. y Bentler, P. M. (1999). "Cutoff criteria for fit indexes in covariance structure analysis: Conventional criteria versus new alternatives", en Structural Equation Modelling, 6: 1-55.

Huebner, E. S. y Gilman, R. (2006). "Students who like and dislike school”, en Applied Research in Quality of Life, 2: 139-150.

Huebner, E. S., Gilman, R., Reschly, A. y Hall, R. (2009). Positive schools. En S. J. Lopez (Ed.), Handbook of positive psychology (2nd ed.). New York: Plenum, 445-455. 
Huebner, E. S., Valois, R. F., Paxton, R. J. y Drane, W. J. (2005). "Middle school students' perceptions of quality of life", en Journal of Happiness Studies, 6: 15-24.

Hui, E. K. P. y Sun, R. C. F. (2010). "Chinese children's perceived school satisfaction: the role of contextual and intrapersonal factors", en Educational Psychology: An International Journal of Experimental Educational Psychology, 30(2): 155-172.

Jöreskog, K. y Sörbom, D. (2003). LISREL 8.54. Structural equation modeling with the Simplis command language. Chicago: Scientific Software International.

Juriševič, M. y Pižorn, K. (2013). "Young Foreign Language Learners' Motivation -A Slovenian Experience", en Porta Linguarum, 19: 179-198.

Kirova, S., Petkovska, B. y Koceva, D. (2012). "Investigation of motivation and anxiety in Macedonia while learning English as a second/foreign language", en Procedia-Social and Behavioral Sciences, 46: 3477-3481.

Khodadady, E. y Khajavy, G. H. (2013). "Exploring the Role of Anxiety and Motivation in Foreign Language Achievement: A Structural Equation Modeling Approach", en Porta Linguarum, 20: 269-286.

Kline, R. B. (2005), Principles and Practice of Structural Equation Modeling (2nd Edition ed.). New York: The Guilford Press.

Levy, R., y Hancock, G. R. (2007). A framework of statistical tests for comparing mean and covariance structure models. Multivariate Behavioral Research, 42, 33-66.

Liu, M., \& Huang, W. (2011). "An exploration of foreign language anxiety and English learning motivation", in Education Research International, 12: 1-8.

Luopa, P., Pietikänen, M. y Jokela, J. (2006). Mistä tukea? Depression, bullying, and truancy among adolescents in Helsinki 1996-2006 (in Finnish). Helsinki: National Institute for Health and Welfare.

Markland, D. (2007). "The golden rule is that there are no golden rules: A commentary on Paul Barrett's recommendations for reporting model fit in structural equation modelling”, en Personality and Individual Differences, 42: 851-858.

McGuirre, B., Parker, L. y Cooper, W. (2001). "Physical Education and language: do actions speak louder than words?", en Physical Eduction \& Sport Pedagogy, 6(2): 101-116.

Miles, J. y Shevlin, M. (2007). "A time and a place for incremental fit indices", en Personality and Individual Differences, 42: 869-874.

Montálvez, M., López, I., y Mariscal, A. (2002). "Desdramaticemos el inglés. Propuesta interdisciplinar: Educación Física (Expresión Corporal) y Lengua Extranjera”, en Retos. Nuevas tendencias en Educación Física, Deporte y Recreación, 1: 29-36.

Nicholls, J. G., Cheung, P.C., Lauer, J. y Patashnick, M. (1989). "Individual differences in academic motivation: Perceived ability, goals, beliefs, and values", en Learning and Individual Differences, 1: 63-84.

Nicholls, J. G., Patashnick, M. y Nolen, S.B. (1985). "Adolescents' theories of education”, en Journal of Educational Psychology, 77: 683-692.

Nunnally, J. C. y Bernstein, I.J. (1995). Teoría psicométrica. Madrid: McGraw-Hill.

Orden de 28 de junio de 2011 por la que se regula la enseñanza bilingüe en los centros docentes de la Comunidad Autónoma de Andalucía. (B.O.J.A), Nº135 del 12 de junio de 2011).

Osterlind, S. J. (1989). Constructing Test Items. Londres: Kluwer Academic Publishers.

Skinner, E., Furrer, C., Marchand, G. y Kinderman, T. (2008). "Engagement and disaffection in the classroom: Part of a larger motivational dynamic?", en Journal of Educational Psychology, 100: 765-781. 
Skutnabb-Kangas, T. y Dunbar, R. (2010). Indigenous Children's Education as Linguistic Genocide and a Crime Against Humanity? A Global View. Kautokeino: Gáldu - Resource Centre for the Rights of Indigenous Peoples.

Spaan, M. (2006). "Test and item specifications development", en Language Assessment Quarterly, 3: 71-79.

Tabachnick, B. G. y Fidell, L. S. (2007). Using Multivariate Statistics (5th ed.). New York: Allyn and Bacon.

Takakura, M., Wake, N. y Kobayashi, M. (2010). "The contextual effect of school satisfaction on health-risk behaviors in Japanese high school students", en Journal of School Health, 80(11): 544-551.

Toledo, I., Rubio, F. D. y Hermosín, M. (2012). "Creencias, rendimiento académico y actitudes de alumnos universitarios principiantes en un programa plurilingüe", en Porta Linguarum, 18: 213-229. 